\title{
Adaptación psicométrica de la Escala de Sentido de Comunidad (SCI-II) en escuelas públicas chilenas
}

\author{
RODRIGO ROJAS-ANDRADE* \\ Universidad Academia de Humanismo Cristiano, Santiago, Chile \\ ORCID: https://orcid.org/0000-0002-6459-6902 \\ PATRICIO CABELLO \\ Universidad San Sebastían, Santiago, Chile \\ ORCID: https://orcid.org/0000-0001-9656-3147 \\ LORETO LEIVA BAHAMONDES \\ Universidad de Chile, Santiago, Chile \\ ORCID: https://orcid.org/0000-0003-0837-3699 \\ NICOLÁS CASTILLO \\ Universidad Academia de Humanismo Cristiano, Santiago, Chile \\ ORCID: https://orcid.org/0000-0001-6796-2317
}

\begin{abstract}
How to quote this article: Rojas-Andrade, R. Cabello, P., Leiva Bahamondes, L. \& Castillo, N. (2019). Adaptación psicométrica de la Escala de Sentido de Comunidad (SCI-II) en escuelas públicas chilenas. Acta Colombiana de Psicología, 22(1), 273-284. doi: http://www.dx.doi.org/10.14718/ ACP.2019.22.1.13
\end{abstract}

Resumen

El presente estudio tuvo como objetivo adaptar la Escala de Sentido de Comunidad (SCI-II) a escuelas chilenas y analizar sus propiedades psicométricas. Se utilizó una muestra compuesta por 2871 estudiantes que cursaban entre $6 .^{\circ}$ de enseñanza básica y $4{ }^{\circ}$ de enseñanza media de la comuna de Calama al norte de Chile. El 52.4\% $\%(n=1506)$ se identificó con el género femenino y la edad promedio de la muestra fue de $14.64(\mathrm{DE}=2.046)$. Como resultados, el análisis factorial exploratorio indicó una solución de tres factores: conectividad escolar, pertenencia valorada y membresía-reciprocidad; la fiabilidad de la escala fue adecuada $(\alpha=.879$ para conectividad escolar, $\alpha=.884$ para pertenencia valorada y $\alpha=.716$ para membresíareciprocidad); y los análisis confirmatorios indicaron índices de ajuste adecuados $(\mathrm{CFI}=.939 ; \mathrm{TLI}=.933)$. Se concluye que el modelo propuesto de la versión adaptada es válido y es utilizable en futuros estudios sobre el sentido de comunidad en el contexto escolar. Al final se discute el valor de este instrumento para las políticas públicas y los procesos de innovación de la educación pública.

Palabras claves: sentido de comunidad, comunidad escolar, validación de instrumentos

\section{Psychometric adaptation of the Sense of Community Index (SCI-II) in Chilean public schools}

\begin{abstract}
This study aimed to adapt the Sense of Community Index (SCI-II) in Chilean schools and to examine its psychometric properties. The validation process was conducted with 2871 students from 6th grade of elementary school to 4th grade of high school in a city of Northern Chile. A $52.4 \%(\mathrm{n}=1506)$ of the sample identified with the female gender and the average age of the sample was $14.64(\mathrm{SD}=2.046)$. An exploratory factor analysis indicated a three-factor solution: school connectivity, valued belongingness, and membership-reciprocity. The reliability of the scale was adequate $(\alpha=.879$ for school connectivity, $\alpha=.884$ for valued belongingness and $\alpha=.716$ for membership-reciprocity), and confirmatory analyzes indicated adequate adjustment rates $(\mathrm{CFI}=.939$; TLI $=.933)$. It was concluded that the proposed model of the adapted version is valid and is appropriate to be used in future studies on the sense of community in the school context. Finally, the article discusses the value of this instrument for public policies and innovation processes of public education.

Keywords: sense of community; school community; instrument validation
\end{abstract}

\footnotetext{
Dr. Escuela de Psicología, Facultad de Ciencias Sociales, Universidad Academia de Humanismo Cristiano, Avenida Condell 343, Providencia, Santiago, Chile. Tel.: 227878203.rrojasa01@docentes.academia.cl Financiación: Fondo PROA.Vice-Rectoría de Investigación y Desarrollo (VIP), Universidad de Chile.
} 


\title{
Adaptaçáo psicométrica da Escala de Sentido de Comunidade (SCI-II) em escolas públicas chilenas
}

\begin{abstract}
Resumo
Este estudo teve como objetivo adaptar a Escala de Sentido de Comunidade (SCI-II) a escolas chilenas e analisar suas propriedades psicométricas. Participaram 2871 estudantes que cursavam entre o $6^{\circ}$ do ensino básico e o $4^{\circ}$ do ensino médio [nomenclatura usada no Chile] da comunidade de Calama, no norte do Chile. $52.4 \%(\mathrm{n}=1506)$ foram identificados com o gênero feminino e idade média da amostra de $14.64(\mathrm{DP}=2.046)$. A análise fatorial exploratória indicou uma solução de três fatores: conectividade escolar, pertencimento valorizado e filiação-reciprocidade. A confiabilidade da escala foi adequada ( $\alpha=.879$ para conectividade escolar, $\alpha=.884$ para pertencimento valorizado e $\alpha=.716$ para filiação-reciprocidade). As análises confirmatórias apresentaram índices de ajuste adequados $(\mathrm{CFI}=.939 ; \mathrm{TLI}=.933)$. Conclui-se que o modelo proposto da versão adaptada é válido e é útil em futuros estudos sobre o sentido de comunidade no contexto escolar. Discute-se o valor desse instrumento para as políticas públicas para os processos de inovação da educação pública.

Palavras-chaves: sentido de comunidade, comunidade escolar, validação de instrumentos
\end{abstract}

\section{Introducción}

Las escuelas no son solo una fuente de transmisión del conocimiento, sino también un espacio comunitario que promueve el encuentro entre personas, el desarrollo de redes de apoyo, la pertenencia y la amistad (Moscardino, Scrimin, Capello \& Altoé, 2010; Puddifoot, 1994; Witten, McCreanor $\&$ Kearns, 2007). En esta línea, el sentido de comunidad ha mostrado ser relevante para el desarrollo socioemocional de los estudiantes, debido a que aquellos estudiantes con alto sentido de comunidad escolar disfrutan más la escuela, están más motivados, mantienen una asistencia óptima, participan en actividades escolares, tienen mejor desempeño académico (Battistich \& Hom, 1997; Elvas \& Moniz, 2010; Pereda, 2003) y son menos propensos a presentar problemas de salud mental (Battistich \& Hom, 1997; Bond et al., 2007).

En este sentido, la conceptualización de la escuela como comunidad combina perspectivas sociológicas y psicológicas que la asumen como un antídoto a las tendencias gerenciales de la sociedad de masas y como un espacio de aprendizaje propio de los seres humanos. Así, se entiende que la comunidad escolar transciende las puertas de la escuela al operar no sobre la base de tareas, reglas y jerarquías, sino sobre los valores, confianzas, expectativas y obligaciones compartidas, así como en aprendizajes sobre el liderazgo comunitario, los vínculos y la gestión colectiva del cambio (Redding, 2001; Watson \& Bogotch, 2016).

Por esta razón, la promoción del sentido de comunidad escolar ha sido uno de los ejes fundamentales de las políticas curriculares y de convivencia en lasúltimas décadas (Baumeister \& Leary, 1995; Frederickson \& Baxter, 2009); sin embargo, son pocos los instrumentos disponibles para medir este constructo, y los que existen se centran especialmente en dimensiones discretas del sentido de comunidad en la escuela, como la conectividad (Goodenow, 1993) o la pertenencia (Rostosky, Owens, Zimmerman \& Riggle, 2003).

En este contexto, contar con instrumentos confiables, válidos, precisos y sensibles a las necesidades de las comunidades escolares es un requisito esencial para que las intervenciones sean exitosas (Dowdy, Furlong, Eklund, Saeki\& Ritchey, 2010).

Uno de los instrumentos más utilizados para medir el sentido de comunidad es el Sense of Community Index-II (SCI-II), basado en el reconocido modelo de McMillan y Chavis (1986), donde se define a través de cuatro dimensiones interdependientes: la pertenencia, la influencia, la satisfacción de necesidades y la conexión emocional compartida (Chavis, Lee \& Acosta, 2008; Chavis \& Pretty, 1999; Peterson, Speer $\&$ McMillan, 2008). En este modelo, la pertenencia se refiere al sentimiento de haber invertido recursos personales en la comunidad y el sentimiento de ser parte de ella; mientras que la influencia se refiere al poder que los miembros ejercen entre sí; la satisfacción de necesidades hace referencia a los valores compartidos por los miembros y los beneficios de pertenecer a la comunidad; $y$ la conexión emocional da cuenta del reconocimiento de lazos compartidos, donde el vínculo es el resultado del contacto positivo y una historia en común (Chavis \& Pretty, 1999). La escala original cuenta con 12 ítems que evalúan los cuatro componentes del modelo, pero en revisiones posteriores se agregaron 12 ítems adicionales con el objetivo de profundizar las dimensiones del sentido de comunidad.

Las investigaciones sobre la estructura factorial de este instrumento han puesto en duda su configuración original (Obst \& White, 2004; Peterson, Speer \& Hughey, 2006), ya que han mostrado empíricamente que la definición de cuatro dimensiones no puede ser concluyente (Flaherty, Zwick \& 
Bouchey, 2014), frente a lo que se han propuesto soluciones de tres (Long \& Perkins, 2003) y de dos factores (Sayer, Beaven, Stringer \& Hermena, 2013). Estos resultados contradictorios también se han observado en estudios iberoamericanos que enfatizan la necesidad de profundizar los fundamentos teóricoempíricos del sentido de comunidad (Sánchez-Vidal, 2001; Sánchez-Vidal, 2009).

Esta inconsistencia es coherente con el consenso sobre que el sentido de comunidad es un constructo multidimensional que conjuga de manera específica los elementos descritos por el modelo McMillan y Chavis (1986) de acuerdo con las características específicas de las comunidades en las que se evalúa (Hombrados, 2011; Jason, Stevens \& Ram, 2015; Wright, 2004). Es necesario considerar esta situación cuando se analiza el sentido de comunidad en la escuela, dada sus especiales particularidades y contextos sociales que se solapan, como el aula, la zona de recreación y la dirección escolar.

Teniendo en cuenta lo anterior, en la presente investigación se buscó adaptar la SCI-II a escuelas públicas chilenas y revisar las propiedades psicométricas en una muestra obtenida a través de una plataforma online con el fin de obtener una versión ajustada a las peculiaridades de las comunidades escolares chilenas y así contar con un instrumento que aporte al diseño intervenciones promocionales y preventivas que permitan fortalecer eficazmente el desarrollo académico y emocional de los estudiantes, así como comprender mejor las características que adquiere el sentido de comunidad cuando se evalúa en comunidades escolares.

\section{Método}

\section{Participantes}

Se aplicó un muestreo no probabilístico intencionado por medio del cual se logró la participación de 2871 estudiantes de 11 establecimientos educacionales municipales de la comuna de Calama, en el norte de Chile, que cubrían los niveles escolares de $6 .^{\circ}$ de enseñanza básica hasta $4 .^{\circ}$ de enseñanza media ${ }^{1}$.

La comuna de Calama es reconocida como la capital minera del país. Su principal producción es la de cobre, cuya exportación sostiene gran parte de la economía chilena. De acuerdo con un estudio de laAsociación de Municipalidades de Chile (2016), Calama se encuentra dentro del segundo grupo de comunas (de cinco) que entrega mejor calidad educativa en el país. Este grupo que se caracteriza por concentrar un $15 \%$ de pobreza y un $17.8 \%$ de población rural. El $44.8 \%$

\footnotetext{
1 El sistema chileno contempla 12 años de enseñanza obligatoria, lo que incluye ocho años de enseñanza básica y cuatro de educación media.
}

de los estudiantes de las comunas asiste a establecimientos municipales, a los cuales se destina el $8.7 \%$ del presupuesto comunal total. La investigación en salud mental infantoadolescente en la comuna ha mostrado que la prevalencia de problemas no difiere significativamente a la del resto del país (Haquin, Larraguibel \& Cabezas, 2004; Rojas-Andrade \& Leiva, 2015).

De los participantes, el $52.4 \%(n=1506)$ se identificaron con el género femenino, mientras que un $0.7 \%(n=20)$ señaló no identificarse con el género femenino ni con el masculino. La edad promedio de la muestra fue de 14.64 años $(\mathrm{DE}=2.046)$ y el $10.60 \%(n=305)$ declaró pertenecer a una nacionalidad distinta a la chilena.

\section{Instrumentos}

Se utilizó la escala de Sentido de Comunidad (SCI-II), que evalúa las dimensiones de pertenencia, influencia, satisfacción de necesidades y conexión emocional compartida a partir del modelo de McMillan y Chavis (1986). Específicamente, el SCI-II consisten en un autoinforme de 24 ítems respondidos en una escala tipo Likert de cuatro opciones de respuesta (con puntajes que van de 0 a 3). Para efectos de esta investigación, se modificó el lenguaje y la redacción de los ítems con el fin de acercarlos a la realidad discursiva de las escuelas; para esto, se contó con la asesoría de tres expertos en educación y psicología escolar, quienes evaluaron la pertinencia del constructo y del lenguaje utilizado para cada ítem.

De este modo, se decidió cambiar la palabra "comunidad" por "escuela" y "conozco" por "ubico", además de dividir el ítem 10 en dos, con tal de explicitar dimensiones simbólicas específicas de las escuelas chilenas asociadas al reconocimiento de características comunes. Así, el ítem original "Las personas que formamos parte de esta comunidad tenemos nuestras propias maneras de vestirnos, como hablar, arreglar nuestras casas, comportarnos" se separó en: "En esta escuela hablamos, nos vestimos y comportamos de una forma parecida que otras personas pueden reconocer" y "En esta escuela tenemos cosas como un himno, insignias, uniformes, estandarte, etc. que otras personas pueden reconocer".

Los índices de confiabilidad obtenidos en investigaciones anteriores del SCI-II han sido adecuados, con valores entre .70 y .90 (Chavis et al., 2008; Peterson et al., 2006; Pretty et al., 1994; Sarriera et al., 2015); no obstante, la estructura factorial del instrumento aún está en discusión por parte de los investigadores. En lo que respecta a la validez de criterio, los estudios han mostrado relaciones adecuadas entre la puntuación total y las variables conductuales, sociales, emocionales y académicas de los miembros de comunidades en las que ha se ha estudiado (Battistich \& Hom, 1997; Bond et al., 2007). 
276

\section{Procedimiento}

Se aplicó el SCII como parte de una batería de instrumentos utilizados para un screening en salud mental escolar en establecimientos educacionales públicos por medio de una plataforma online, cuyo proceso de implementación siguió el siguiente curso: (1) contacto con autoridades municipales; (2) invitación a establecimientos; (3) capacitación presencial, supervisión y tutoría en línea a los aplicadores mediante mensajería instantánea para resolver dudas de implementación; (4) envío y recepción de consentimientos negativos (expresión explícita del deseo de no participar); (5) firma de asentimiento de los estudiantes; (6) aplicación supervisada del instrumento mediante una plataforma digital abierta y gratuita que se encontraba disponible en los laboratorios de informática de los establecimientos; y (7) análisis y envío de informe por niño, curso y establecimiento.

\section{Aspectos éticos}

Se contó la aprobación del comité de ética de una de las universidades patrocinadoras del estudio, con el consentimiento de padres y el asentimiento de niños, niñas y adolescentes participantes. Para esto último, se utilizaron cartas de asentimiento para los estudiantes y de "consentimiento negativo" para los padres; es decir, que aquellos que no aceptaron que sus hijos o hijas participaran en la investigación tuvieron la oportunidad de expresarlo por escrito.

Toda la información recabada fue confidencial, aunque no anónima. Si bien se obtuvieron los números de identificación de cada niño, niña y adolescente, estos datos se disociaron de los nombres y solo se utilizaron en conjunto para organizar el material para los informes conforme a las necesidades del proceso de screening convenido con los centros. En este mismo marco, como forma de devolución de información a las comunidades escolares, al final del proceso se entregaron informes tanto a nivel general como por cada niño, niña y adolescente. De esta manera, la información personal de los y las estudiantes fue tratada de manera tal que no fuera posible que terceros tuvieran acceso directo a ella.

\section{Diseño de análisis de datos}

Se siguió un diseño instrumental (Ato, López \& Benavente, 2013) y se utilizaron como técnicas de análisis el Análisis Factorial Exploratorio (AFE) y Análisis Factorial Confirmatorio (AFC) asistidos a través de los software SPSS-IBM 22.0 y MPLUS 7. Para cada uno de los análisis se utilizaron submuestras independientes extraídas aleatoriamente de la muestra general (1426 para AFE y 1445 para AFC).

El AFE analiza la estructura estadística de las respuestas de un instrumento y es utilizado para determinar la estructura interna de números generalmente grandes de factores que subyacen los datos, permitiendo que todos los factores estén correlacionados debido a la saturación de todos los indicadores con todos los factores. Este procedimiento es muy útil en los casos donde el investigador desconoce de antemano la estructura que se puede esperar de las respuestas de la muestra y, dado el escaso acuerdo de la configuración del SCI-II, se decidió utilizarlo para identificar la estructura factorial empírica de la matriz de datos obtenida.

Por otra parte, elAFC se utiliza para determinar si el número de factores obtenidos se ajustan con los que corresponden a la de un postulado o teoría previa sobre los datos, con el fin de confirmar empíricamente la estructura conceptual establecida a priori. En este caso, este procedimiento se utilizó para calcular indicadores de bondad y ajuste, y compararlos con aquellos que surgían de las propuestas factoriales originales. Se consideró que el modelo que mostrara mejores valores de ajuste sería el que mejor reflejaría la estructura empírica del instrumento en la población estudiada.

Como primera fase de análisis, se revisaron las distribuciones de los ítems y la matriz de correlaciones, y se calculó el índice de adecuación muestral de Kaiser-Meyer-Olkin (KMO, cuyos valores cercanos a uno indican adecuación al modelo de AFE), el contraste de esfericidad de Barlett (cuya significación menor al 5 \% señala la ausencia de una matriz de identidad) y las medidas de suficiencia del muestreo (MSA, cuyos valores cercanos a uno dan cuenta de que los ítems pueden ser predichos sin error por otros ítems) para evaluar la pertinencia de realizar un análisis factorial.

Tanto para el AFE como para el AFC se estimaron los factores considerando el método de los mínimos cuadrados generalizados WLSMV (Weighted Least Squares Means and Variance Adjusted), debido al nivel ordinal de medición y el no cumplimiento del supuesto de normalidad de los ítems.

A continuación, para evaluar la bondad de ajuste de los modelos desprendidos del AFE y AFC se tomaron en cuenta los indicadores $\chi 2 / g 1$ (chi-cuadrado/grado de libertad), CFI (compared fixed index), TLI(Tuker-Lewis Index), RMSEA(Root Mean Square Error of Approximation) y SRMR (Standardized Root Mean Square Residual) por ser reconocidos como indicadores altamente robustos para la estimación de modelos factoriales (Bandalos \& Finney, 2010). Específicamente, el $\chi 2 / g 1$ es una medida de ajuste absoluto, donde proporciones menores a dos o tres son aceptables, sin embargo, el contraste de este índice se hace más sensible a medida que aumentan los parámetros, por lo que se hace recomendable analizarlo en conjunto con otras medidas de ajuste (Tanaka, 1998); el CFI y el TLI son medidas de ajuste incremental, donde valores cercanos a uno se consideran indicativos de buen ajuste; el RMSEA es una medida de parsimonia, donde valores menores a .08 se consideran adecuados; y el SRMR es una medida de 
ajuste absoluto, donde valores menores .08 son considerados indicativos de buen ajuste global (Bandalos \& Finney, 2010).

Finalmente, se calculó la confiabilidad de cada dimensión a través del $\alpha$ de Cronbach y se estimaron descriptivos por dimensión.

\section{Resultados}

A continuación se reporta, en primer lugar, el análisis de cada uno de los ítems y el análisis de la matriz de correlaciones entre ellos; posteriormente, se da cuenta del análisis de la dimensionalidad de la escala a través de AFE y AFC; y, por último, se informa de los resultados de los análisis de confiabilidad y se presentan estadísticos descriptivos de las escalas.
Análisis de los ítems

En la Tabla 1 se da cuenta de los descriptivos de cada ítem de la escala SCI-II adaptada a escuelas chilenas. El promedio general de los ítems fue de $1.835(\mathrm{DE}=.917)$, y los ítems que presentaron medias cercanas a los tres puntos fueron los ítems 1, 4, 11, 21, 23 y 24. De ellos, destacan el ítem 11 ("En esta escuela tenemos cosas como un himno, insignias, uniformes, estandarte, etc. que otras personas pueden reconocer") y el ítem 21 ("Paso mucho tiempo con otras personas de la escuela y disfruto estando con ellos"). Respecto a la distribución de los ítems, se realizaron pruebas Kolmogorov-Smirnov por medio de las cuales se observó que ninguno de ellos se ajusta a los parámetros de una distribución normalidad. Los estadísticos descriptivos de asimetría y apuntamiento también se presentan en la Tabla 1.

Tabla 1.

Descriptivos de los items del SCI-II adaptado a escuelas chilenas

\begin{tabular}{|c|c|c|c|c|c|c|}
\hline & Ítems & Media & $\mathrm{DE}$ & A & $\mathrm{k}$ & $\mathrm{D}$ \\
\hline 1 & En esta escuela yo puedo satisfacer necesidades que son importantes para mí. & 2.010 & .831 & -.507 & -.351 & $.248 *$ \\
\hline 2 & Todos en la escuela valoramos las mismas cosas. & 1.369 & .885 & .153 & -.694 & $.236^{*}$ \\
\hline 3 & Esta escuela ha sido capaz de satisfacer las necesidades de sus integrantes. & 1.980 & .814 & -.414 & -.425 & $.249 *$ \\
\hline 4 & Ser parte de esta escuela me hace sentir bien. & 2.228 & .894 & -.959 & -.029 & $.287 *$ \\
\hline 5 & Cuando tengo un problema, puedo conversarlo con personas de esta escuela. & 1.750 & 1.100 & -.292 & -1.261 & $.207 *$ \\
\hline 6 & Las personas en esta escuela tienen necesidades, prioridades y metas parecidas. & 1.956 & .857 & -.451 & -.493 & $.243^{*}$ \\
\hline 7 & Puedo confiar en las personas de esta escuela. & 1.617 & .990 & -.146 & -1.012 & $.209 *$ \\
\hline 8 & Ubico a la mayoría de las personas de esta escuela. & 1.796 & .905 & -.216 & -.827 & $.213^{*}$ \\
\hline 9 & Casi todas las personas de esta escuela me ubican. & 1.394 & .882 & .152 & -.682 & $.239 *$ \\
\hline 10 & $\begin{array}{l}\text { En esta escuela hablamos, nos vestimos y comportamos de una forma parecida } \\
\text { que otras personas pueden reconocer. }\end{array}$ & 1.747 & .908 & -.292 & -.702 & $.241 *$ \\
\hline 11 & $\begin{array}{l}\text { En esta escuela tenemos cosas como un himno, insignias, uniformes, estandarte, } \\
\text { etc. que otras personas pueden reconocer. }\end{array}$ & 2.639 & .681 & $-1,962$ & 3.380 & $.439 *$ \\
\hline 12 & Pongo mucho tiempo y esfuerzo en ser parte de esta escuela. & 1.956 & .837 & -.486 & -.326 & $.259 *$ \\
\hline 13 & Pertenecer a esta escuela es una parte de quien soy. & 1.868 & .981 & -.425 & -.872 & $.215^{*}$ \\
\hline 14 & Ser como las personas de esta escuela es importante para mí. & 1.408 & 1.030 & .061 & -1.170 & $.193^{*}$ \\
\hline 15 & Lo que hacemos en esta escuela tiene un impacto en otras partes. & 1.860 & .885 & -.380 & -.597 & $.243 *$ \\
\hline 16 & Me preocupa lo que otras personas de la escuela piensen de mí. & 0.974 & 1.080 & .687 & -.924 & $.283 *$ \\
\hline 17 & Tengo influencia en las cosas que pasan en mi escuela. & 1.145 & .931 & .326 & -.841 & $.211 *$ \\
\hline 18 & Si hay un problema en esta escuela, las personas lo pueden resolver. & 1.955 & .923 & -.457 & -.742 & $.213 *$ \\
\hline 19 & Esta escuela tiene buenos líderes. & 1.928 & .980 & -.491 & -.831 & $.210^{*}$ \\
\hline 20 & Es muy importante para mí ser parte de esta escuela. & 1.907 & .992 & -.470 & -.879 & $.208^{*}$ \\
\hline 21 & Paso mucho tiempo con otras personas de la escuela y disfruto estando con ellos. & 2.263 & .890 & -.987 & .018 & $.305^{*}$ \\
\hline 22 & Espero sentirme parte de esta escuela por mucho tiempo. & 1.957 & 1.010 & -.555 & -.839 & $.226^{*}$ \\
\hline 23 & $\begin{array}{l}\text { Los miembros de esta escuela han compartido cosas buenas y cosas malas, como } \\
\text { celebraciones o desastres. }\end{array}$ & 2.270 & .808 & -.869 & .032 & $.284 *$ \\
\hline 24 & Siento que el futuro de esta escuela será bueno. & 2.244 & .881 & -.948 & .001 & $.293 *$ \\
\hline 25 & Los integrantes de esta escuela se cuidan y protegen. & 1.662 & .939 & -.199 & -.845 & $.227 *$ \\
\hline
\end{tabular}

Nota. En negrilla aparecen los valores más altos; $\mathrm{DE}=$ desviación estándar; $\mathrm{A}=$ asimetría; $\mathrm{K}=$ curtosis; $\mathrm{D}=$ estadístico de contraste para la prueba Kolmogorov-Smirnov; ${ }^{*} p<.05$. 


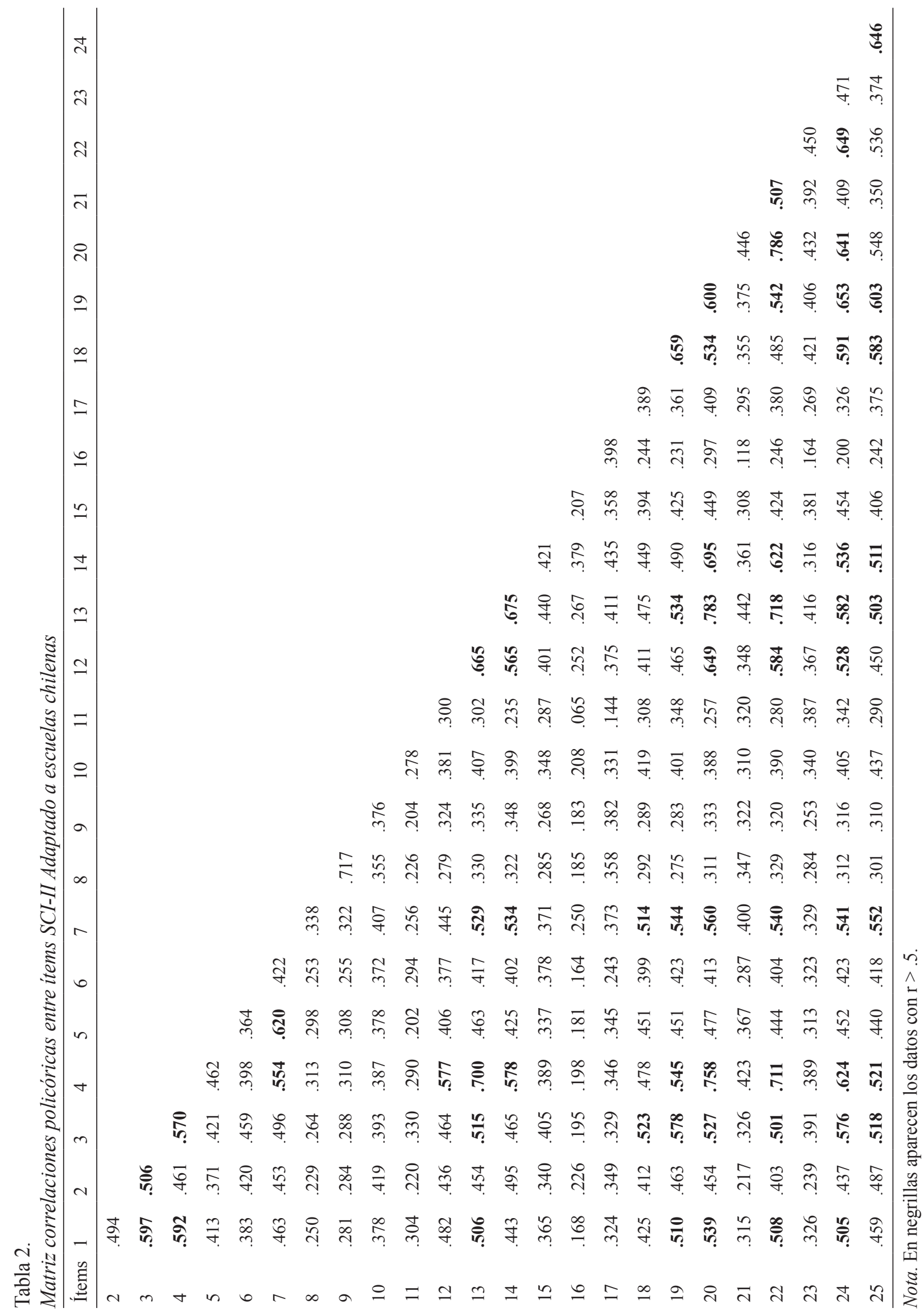




\section{Correlaciones entre items}

En la Tabla 2 se muestra la matriz de correlaciones policóricas, donde se puede observar que la totalidad de ellos están correlacionados de manera adecuada, aunque aquellos que tienen correlaciones altas, de los que destacan las correlaciones entre los ítems $13-20(\mathrm{r}=.783)$ y $19-22$ $(\mathrm{r}=.786)$, refieren a la dimensión de pertenencia en la escuela.

Análisis de dimensionalidad: AFE y AFC

Análisis factorial exploratorio.

El índice KMO fue de .959, lo que evidenció la pertinencia de realizar el análisis factorial entre los 35 ítems del SCI-II; mientras que el contraste de esfericidad de Barlett mostró valores adecuados $\left(x_{(465)}^{2}=124106.959 ; \mathrm{p}>.001\right)$ y los MSA se resultaron en un rango de .873 y .982 , lo que se considera satisfactorio para el análisis propuesto.

Los resultados del AFE se muestran en la Tabla 3, donde se puede observar que el modelo de tres factores es el que presenta los mejores indicadores de ajuste entre los modelos testeados, con índices de ajuste absoluto adecuados $(\chi 2 / \mathrm{gl}=5.78 ; \mathrm{SRMR}=.036 ; \mathrm{RMSEA}=.058) \mathrm{e}$ índices de ajuste comparativos aceptables, cercanos a uno $(\mathrm{CFI}=.970 ; \mathrm{TLI}=.960)$. Si bien los modelos descartados muestran índices adecuados, se decidió mantener el de tres factores tanto por motivos teóricos (intentando mantener la estructura original) como por la competencia entre los indicadores respecto a sus modelos rivales y el ajuste a la distribución empírica de los datos.

Tabla 3.

Indicadores de Bondad del ajuste del AFE para el SCI-II adaptado a escuelas chilenas

\begin{tabular}{ccccc} 
Modelo & $\chi^{2}$ & gl & $\chi 2 /$ gl CFI TLI RMSEA SRMR \\
\hline
\end{tabular}

\begin{tabular}{lllllll}
\hline Un factor & $4189^{*}$ & 275 & 15.23 & .891 .881 & .100 & .062
\end{tabular}

Dos factores $2100.478 * 251 \quad 8.36 .949 .939 \quad .072 \quad .047$

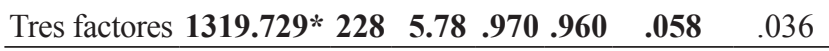

Nota. En negrita aparecen los indicadores con mejor ajuste; $* \mathrm{p}<.001$.

Para analizar la configuración de los factores, y con el fin de mejorar la interpretación del modelo y favorecer la solución factorial local, se utilizó el método de rotación oblicua GEOMIN (G) (Hattori. Zhang \& Preacher, 2017), mientras que, para ubicar los ítems en los distintos factores, se utilizaron criterios tanto teóricos -para resguardar la teoría de McMillan y Chavis (1986) - como estadísticos - para destacar las significancias estadísticas de las cargas factoriales $(\mathrm{p}<.05)-$.
Tabla 4.

Cargas de cada item para los tres factores

\begin{tabular}{|c|c|c|c|}
\hline Ítems & Factor 1 & Factor 2 & Factor 3 \\
\hline 1 & $.515 *$ & $.239 *$ & -.091 \\
\hline 2 & $.561 \%$ & $.079 *$ & .008 \\
\hline 3 & $.775 \%$ & .016 & $-.098 *$ \\
\hline 4 & $.189 *$ & $.694 \%$ & $-.024 *$ \\
\hline 5 & $.537 *$ & $.053^{*}$ & $.124 *$ \\
\hline 6 & $.568 \%$ & -.012 & $.040 *$ \\
\hline 7 & $.607 *$ & $.083 *$ & $.090 *$ \\
\hline 8 & -.007 & .001 & $.859 *$ \\
\hline 9 & .003 & .058 & $.804 *$ \\
\hline 10 & $.511 *$ & .066 & $.253 *$ \\
\hline 11 & $.483 *$ & -.062 & $.055 \%$ \\
\hline 12 & .092 & $.678 *$ & $.018 *$ \\
\hline 13 & $-.016^{*}$ & $.888 *$ & $.009 *$ \\
\hline 14 & .094 & $.654 \%$ & $.084 *$ \\
\hline 15 & $.406 *$ & $.163^{*}$ & $.075 \%$ \\
\hline 16 & .051 & $.315^{*}$ & $.190 \%$ \\
\hline 17 & $.142 *$ & $.255^{*}$ & $.279 *$ \\
\hline 18 & $.860 \%$ & $-.140 *$ & .007 \\
\hline 19 & $.833 *$ & -.013 & $-.063^{*}$ \\
\hline 20 & -.011 & $.920 *$ & -.006 \\
\hline 21 & $.282 *$ & $.207 *$ & $.187 *$ \\
\hline 22 & .093 & $.775 *$ & .010 \\
\hline 23 & $.460 *$ & $.084^{*}$ & $.102 *$ \\
\hline 24 & $.687 *$ & $.182^{*}$ & $-.060 *$ \\
\hline 25 & $.710 *$ & .005 & .026 \\
\hline
\end{tabular}

Nota. En negrita aparecen las cargas correspondientes a la conformación de ítems de los tres factores.

Como se puede observar en la Tabla 4, los ítems que más cargan en el Factor 1 fueron el 18 ("Si hay un problema en esta escuela, las personas lo pueden resolver"; $\mathrm{G}=.860$ ) y el 19 ("Esta escuela tiene buenos lideres"; $\mathrm{G}=.833$ ); mientras que los que cargaron en el Factor 2 fueron el ítem 13 ("Pertenecer a esta escuela es una parte de quien soy"; $\mathrm{G}=.888$ ) y el 20 ("Es muy importante para mí ser parte de esta escuela"; $\mathrm{G}=.920$ ); $y$, finalmente, los que presentaron mayor carga factorial en el Factor 3 fueron el ítem 8 ("Ubico a la mayoría de las personas de esta escuela"; $\mathrm{G}=.859$ ) y el 9 ("Casi todas las personas de esta escuela me ubican;" $\mathrm{G}=.804)$.

Con base en la conformación de ítems, se decidió nombrar al Factor 1 "Conectividad escolar", debido a que incluye elementos asociados a la satisfacción de necesidades compartidas y está relacionado con la capacidad de poner en práctica liderazgos para la solución de problemas, el sentido de seguridad, la proyección de la comunidad escolar, la articulación de la confianza y los objetivos comunes, al 
brindar apoyo social y conexión emocional. Esto es consistente con la definición internacional de dicho constructo (Goodenow, 1993).

Asimismo, se decidió nombrar al Factor "Pertenencia valorada", debido a que se refiere a la sensación de ser parte de la comunidad y el aporte afectivo que la comunidad tiene para sí mismo, lo que incluye tanto la sensación de una relación estable con la comunidad que se proyecta en el tiempo. Este factor ha sido asociado al bienestar emocional y académico de los estudiantes (Rostosky, Owens. Zimmerman \& Riggle. 2003; Israelashvili. 1997).

Y, por último, se decidió nombrar al Factor 3 "Membresíareciprocidad", gracias a que se refiere a las relaciones de reconocimiento mutuo, la sensación de ser considerado y la validación entre integrantes de la comunidad escolar. Se decidió mantener el ítem 15 en este factor a pesar de su baja carga por la importancia conceptual en el modelo y por su carga final en el AFC.

\section{Análisis factorial confirmatorio}

Al comparar los indicadores de AFC del modelo original (cuatro factores) con el modelo propuesto de tres factores (véase Tabla 5), se puede observar que este último refleja con mayor bondad la distribución empírica de los datos locales. En la Figura 1 se muestran los pesos de regresión del modelo seleccionado, donde se puede observar que las tres dimensiones muestran una alta relación, sobre todo entre la Pertenencia valorada y la Conectividad escolar.

Con respecto a los ítems que más cargan en cada dimensión, destacan el ítem 24 para Conectividad escolar ("Siento que el futuro de esta escuela será bueno"), el ítem 13 para Pertenencia valorada ("Pertenecer a esta escuela es una parte de quien soy") y el ítem 15 para Membresíareciprocidad ("Lo que hacemos en esta escuela tiene un impacto en otras partes").

\section{Análisis de confiabilidad y estadísticos descriptivos}

Los coeficientes de confiabilidad se calcularon con el $\alpha$ de Cronbach, y se observó que las tres dimensiones presentaron una alta consistencia: $\alpha=.879$ para Conectividad escolar, $\alpha=.884$ para Pertenencia valorada y $\alpha=.716$ para

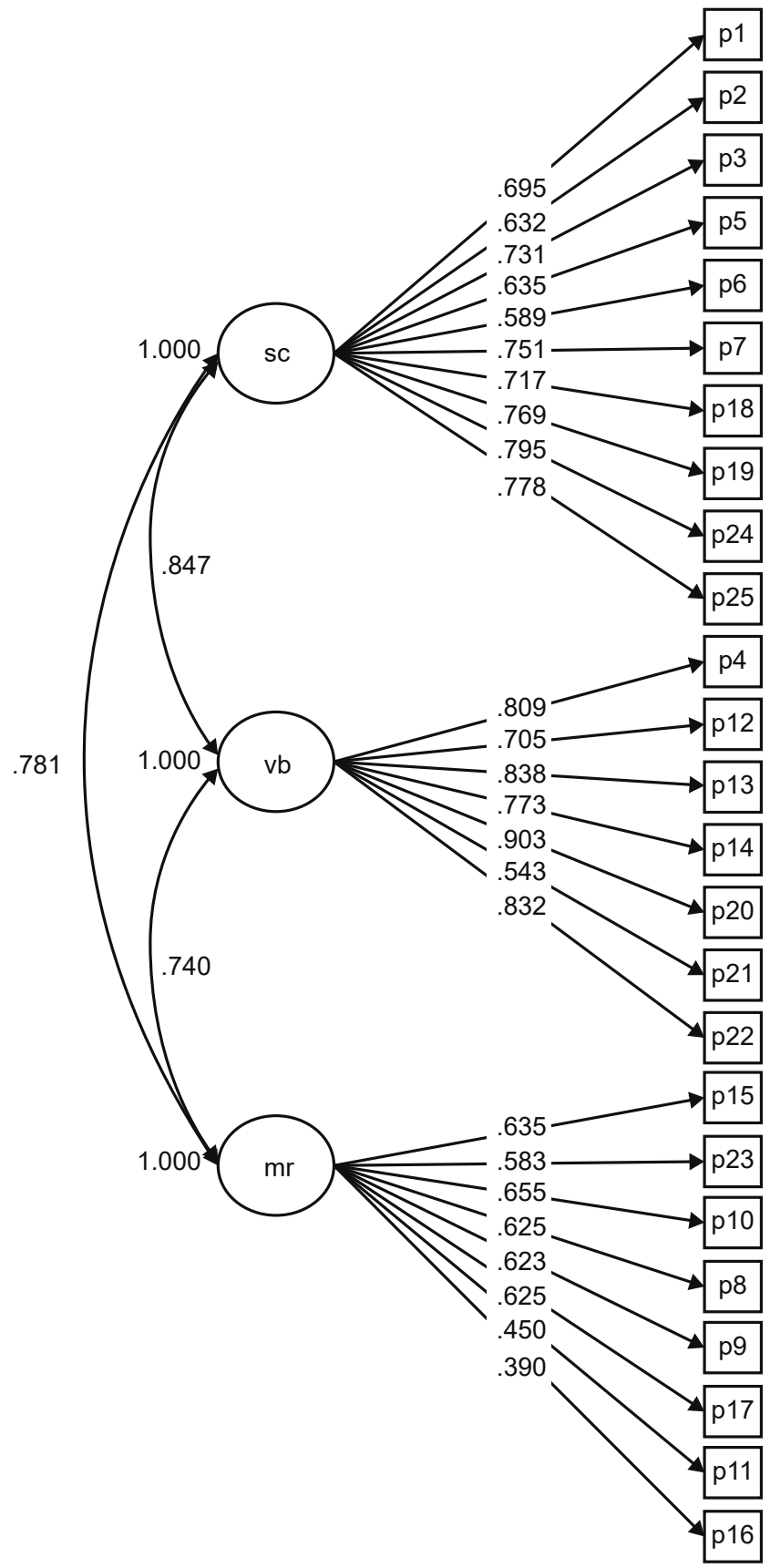

Figura 1. Modelo de tres factores para el SCI-II adaptado a las escuelas chilenas. ce $=$ Conectividad escolar; $\mathrm{pv}=$ Pertenencia valorada; $\mathrm{mb}=$ Membresía-reciprocidad.

Tabla 5.

Comparación de indicadores de ajuste entre el modelo original y el SCI-II adaptado a escuelas chilenas

\begin{tabular}{ccccccc}
\hline Modelo & $\chi^{2}$ & $\mathrm{gl}$ & $\chi 2 / \mathrm{gl}$ & $\mathrm{CFI}$ & TLI & RMSEA \\
\hline Modelo McMillan y Chavis (cuatro factores) & $21237.393^{*}$ & 269 & 78.94 & .410 & .342 & .232 \\
Modelo de tres factores propuesto & $2428.755^{*}$ & 272 & 8.92 & .939 & .933 & .074 \\
\hline
\end{tabular}

Nota. En negrillas aparecen los indicadores con mejor ajuste; ${ }^{*} \mathrm{p}<.001$. 
Membresía-reciprocidad. El puntaje final de las dimensiones del sentido de comunidad se obtuvo de la suma de los ítems identificados para cada uno de ellos, y, para evaluar su distribución, se realizaron pruebas Kolmogórov-Smirnov, por medio de las cuales se observó que ninguna de ellas sigue una distribución normal. Los estadísticos descriptivos de las dimensiones se muestran en la Tabla 6 .

Adicionalmente, se utilizó la prueba no paramétrica Kruskal-Wallis para comparar las puntuaciones de acuerdo con los grupos de edad, y en ella se encontró una diferencia significativa con respecto a las cohortes de edad tanto para la Conectividad escolar $\left(x_{(3)}^{2}=452.255 ; \mathrm{p}<.001\right)$, Pertenencia Valorada $\left(x_{(3)}^{2}=379.851 ; \mathrm{p}<.001\right)$ como para la Membresía-reciprocidad $\left(x_{(3)}^{2}=259.313 ; \mathrm{p}<.001\right)$, donde se evidencian valores menores en los grupos de más edad. Los percentiles según rango etario se muestran en la Tabla 7.

Con respecto al género, por medio de la prueba $U$ de Mann-Whitney se encontró que no existen diferencias significativas entre hombres y mujeres en ninguna de las dimensiones de sentido de comunidad $(U=975747.5$; $p>.05$ para Conectividad escolar; $U=998203 ; p>.05$ para Pertenencia valorada $y ; U=1002160.5 ; y p>.05$ para Membresía-reciprocidad). Se excluyó del análisis aquellos que declaraban pertenecer a otro género debido al pequeño tamaño del grupo.

\section{Discusión}

La presente investigación tuvo como objetivo adaptar la escala SCI-II al contexto de las comunidades escolares públicas chilenas. Como resultado de la validación estadística del instrumento, se revelan adecuados indicadores de validez y fiabilidad en términos de la composición factorial, consistencia interna e, incluso, en las propiedades de los ítems. Según los resultados, la escala incorpora la Conectividad escolar, la Pertenencia valorada y la Membresía-reciprocidad como las dimensiones que subyacen el sentido de comunidad de los niños, niñas y adolescentes en las escuelas.

En esta versión, la escala adaptada presenta una estructura dimensional diferente a la propuesta original, ya que las dimensiones que surgieron de los análisis de la medida

Tabla 6.

Estadisticos descriptivos de las escalas del SCI-II adaptado a escuelas chilenas

\begin{tabular}{lcccccccc}
\hline \multirow{2}{*}{ Dimensiones } & \multirow{2}{*}{ Media } & \multirow{2}{*}{ DE } & \multirow{2}{*}{ A } & \multirow{2}{*}{ K } & \multirow{2}{*}{ D } & \multicolumn{3}{c}{ Percentiles } \\
& & & & & P25 & P50 & P75 \\
\hline Conectividad escolar & 18.471 & 6.388 & -.351 & -.481 & $.069^{*}$ & 14.0 & 19.0 & 24.0 \\
Pertenencia valorada & 13.587 & 5.114 & -.540 & -.479 & $.101^{*}$ & 10.0 & 14.0 & 18.0 \\
Membresía-reciprocidad & 13.825 & 4.137 & -.104 & -.104 & $.061^{*}$ & 11.0 & 14.0 & 17.0 \\
\hline
\end{tabular}

Nota $. \mathrm{DE}=$ desviación estándar; $\mathrm{A}=$ asimetría $\mathrm{K}=$ curtosis; $\mathrm{D}=$ estadístico de contraste para la prueba Kolmogórov-Smirnov; ${ }^{*} \mathrm{p}<.05$.

Tabla 7.

Percentiles de las escalas del SCI-II adaptado a escuelas chilenas según el rango etario

\begin{tabular}{|c|c|c|c|c|c|c|c|}
\hline \multirow{2}{*}{ Dimensiones } & \multicolumn{7}{|c|}{ Percentiles } \\
\hline & 5 & 10 & 25 & 50 & 75 & 90 & 95 \\
\hline \multicolumn{8}{|c|}{ Conectividad escolar } \\
\hline 10 a 12 años & 11.00 & 16.00 & 20.00 & 24.00 & 27.00 & 29.00 & 30.00 \\
\hline 13 a 14 años & 9.00 & 11.00 & 16.00 & 21.00 & 24.00 & 27.00 & 28.00 \\
\hline 15 a 16 años & 7.00 & 9.00 & 12.00 & 17.00 & 21.00 & 25.00 & 26.40 \\
\hline 17 y más años & 5.00 & 8.00 & 11.00 & 16.00 & 20.00 & 24.00 & 25.80 \\
\hline \multicolumn{8}{|c|}{ Pertenencia valorada } \\
\hline 10 a 12 años & 7.50 & 11.00 & 15.00 & 18.00 & 20.00 & 21.00 & 21.00 \\
\hline 13 a 14 años & 5.00 & 7.00 & 11.00 & 15.00 & 18.00 & 20.00 & 21.00 \\
\hline 15 a 16 años & 3.00 & 5.00 & 9.00 & 13.00 & 16.00 & 19.00 & 20.00 \\
\hline 17 y más años & 3.00 & 5.00 & 8.00 & 12.00 & 16.00 & 18.00 & 20.00 \\
\hline \multicolumn{8}{|c|}{ Membresía-reciprocidad } \\
\hline 10 a 12 años & 9.00 & 11.00 & 13.00 & 16.00 & 19.00 & 21.00 & 22.00 \\
\hline 13 a 14 años & 8.00 & 9.00 & 12.00 & 15.00 & 17.00 & 19.00 & 21.00 \\
\hline 15 a 16 años & 6.00 & 8.00 & 10.00 & 13.00 & 15.00 & 18.00 & 19.00 \\
\hline 17 y más años & 6.00 & 8.00 & 10.00 & 13.00 & 15.00 & 18.00 & 19.00 \\
\hline
\end{tabular}


no se agruparon de acuerdo con lo esperado; no obstante, la adaptación conserva la estructura de los ítems basada en la conceptualización del sentido de comunidad (McMillan \& Chavis, 1986). Estos resultados son consistentes con los reportados en otras investigaciones que proponen que la configuración factorial del SCI-II no se ajusta a la propuesta original, por lo que resulta necesario revisar y actualizar la base teórica del sentido de comunidad (Flaherty et al., 2014; Long \& Perkins, 2003; Sayer et al., 2013).

Las dimensiones subyacentes del constructo de sentido de comunidad derivadas del análisis factorial exploratorio fueron construidas considerando como referencia la literatura. Así, el primer factor fue denominado "Conectividad escolar", por enfatizar la percepción de apoyo y preocupación mutua entre los miembros de una escuela (Goodenow, 1993); el segundo se denominó "Pertenencia valorada" (Rostosky et al., 2003; Israelashvili, 1997), por su énfasis en la valoración emocional positiva que se le otorga a la pertenencia a la escuela; y, el último, se denominó "Membresía-reciprocidad", por destacar la capacidad de influencia y valoración mutua que tienen los miembros de una comunidad escolar.

En este contexto, los resultados de la investigación sugieren que, para experimentar un fuerte sentido de comunidad escolar, los niños, niñas y adolescentes deben percibir, principalmente, los beneficios de ser un miembro valorado en la comunidad, además de recibir apoyo social por parte de los miembros.

Asimismo, con respecto a las propiedades psicométricas, los resultados obtenidos en los análisis demuestran que el instrumento es una medida fiable dada la buena consistencia interna de las dimensiones y los factores que la conforman $(\alpha$ de Cronbach $=.879$ para Conectividad escolar, $\alpha=.884$ para Pertenencia valorada y $\alpha=.716$ para Membresía-reciprocidad), y que cuenta con altos índices de ajuste $(\mathrm{CFI}=.939$; $\mathrm{TLI}=.933)$. Por esto, se considera que la escala presenta buen rendimiento en la muestra y que puede ser utilizada en futuras investigaciones con niños, niñas y adolescentes en comunidades escolares.

Debido a lo mencionado, se asevera que la escuela como comunidad es particularmente importante como un medio social para la conexión con el contexto educativo de los niños y niñas, ya que se ha convertido en una instancia y espacio clave para construir los factores de protección para los resultados educativos positivos y menores tasas de comportamientos de riesgo en su salud mental y bienestar (Sayer et al., 2013). De este modo, los beneficios que aporta un fuerte sentido de comunidad a los niños y niñas en la escuela son múltiples, al mismo tiempo que contribuyen a una mayor satisfacción del profesorado (Ariza, QuevedoBlasco \&, Buela-Casal, 2014; Wright, 2004).
Por otra parte, una limitación de esta investigación es la especificidad territorial de la muestra utilizada, por lo que se sugiere continuar estudios en comunidades escolares para comprobar el funcionamiento del instrumento adaptado en otros territorios o contextos (por ejemplo, en escuelas de diferentes regiones). De igual forma, se recomienda indagar en trabajos posteriores sobre los índices de vulnerabilidad y desempeño académico, debido a que podrían ser factores relevantes para discutir las diferencias en la configuración del sentido de comunidad; además de analizar la invarianza configural del instrumento evaluando si la estructura factorial es similar entre distintas subpoblaciones asociadas al género, la edad o el nivel socioeconómico.

Por último, es importante mencionar que el presente es el primer instrumento adaptado y validado en el contexto escolar latinoamericano que mide de manera integral el constructo de sentido de comunidad, y que, además, se aplica por medio de una plataforma digital. Contar con este instrumento validado constituye un aporte a la comprensión de factores no académicos que pueden incidir en la salud mental y el desempeño académico de estudiantes, aspecto crucial para la discusión de las políticas públicas en educación.

\section{Referencias}

Ariza, T., Quevedo-Blasco, R., \& Buela-Casal, G. (2014). Satisfaction of social and legal sciences teachers with the introduction of the European Higher Education Area. European Journal of Psychology Applied to Legal Context, (6), 9-16. doi: 10.5093/ejpalc2014a2

Asociación de Municipalidades de Chile (2016). Calidad de la Educación en las Comunas de Chile. Recuperado de http://www.amuch.cl/pdf/estudio_calidad_de_la_educa cion_2015.pdf

Ato, M., López, J., \& Benavente, A. (2013). Un sistema de clasificación de los diseños de investigación en psicología. Anales de Psicología, 29(3), 1038-1059. doi: 10.6018/ana lesps.29.3.178511

Bandalos, D., \& Finney, S. (2010). Factor analysis: Exploratory and confirmatory. En G. R. Hancock \& R. O. Mueller (Eds.), The reviewer's guide to quantitative methods in the Social Sciences (pp. 93-114). New York: Routledge.

Battistich, V., \& Hom, A. (1997). The relationship between student's sense of their school as a community and their involvement in problem behaviors. American Journal of Public Health, 87, 1997-2001. doi: 10.2105/ AJPH.87.12.1997

Baumeister, R., \& Leary, M. (1995). The need to belong: desire for interpersonal attachments as a fundamental human 
motivation. Psychological Bulletin, 117, 497-529. doi: 10.1037/0033-2909.117.3.497

Bond L., Butler H., Thomas L., Carlin J., Glover S., Bowes G., \& Patton G. (2007). Social and school connectedness in early secondary school as predictor's of late teenage substance use, mental health, and academic outcomes. Journal of Adolescent Health, 40(4), 357. doi:10.1016/j.jado] health.2006.10.D13

Chavis, D., \& Pretty, G. (1999). Sense of community: Advances in measurement and application. Journal of Community Psychology, 27(6), 635-642. doi: https://doi. org/10.1002/(SICI)1520-6629(199911)27:6<635::AIDJCOP1>3.0.CO;2-F

Chavis, D., Lee, K., \& Acosta J. (2008). The Sense of Community (SCI) Revised: The Reliability and Validity of the SCI-II. Paper presented at the 2nd International Community Psychology Conference, Lisboa, Portugal.

Dowdy, E., Furlong, M., Eklund, K., Saeki, E., \& Ritchey, K. (2010). Screening for mental health and wellness: Current school-based practices and emerging possibilities. En B. Doll, W. Pfohl, \& J. Yoon (Eds.), Handbook of youth prevention science (pp. 70-95). New York, NY: Routledge.

Elvas, S., \& Moniz, M. (2010). Sentimento de comunidade, qualidade e satisfação de vida. Análise Psicológica, 28(3), 451-464. doi: 10.14417/ap.312

Flaherty, J., Zwick, R., \& Bouchey, H. A. (2014). Revisiting the sense of community index: A confirmatory factor analysis and invariance test. Journal of Community Psychology, 42(8), 947-963. doi: https://doi.org/10.1002/jcop.20027

Frederickson, N., \& Baxter, J. (2009). Measures of Children's Mental Health \& Psychological Wellbeing series: A portfolio for education and health professionals. Belonging, London: GL Assessment.

Goodenow, C. (1993). Classroom belonging among early adolescent students: Relationships to motivation and achievement. Journal of Early Adolescence, 13, 21-43. doi: 10.1177/0272431693013001002

Haquin, C., Larraguibel, M., \& Cabezas, J. (2004). Factores protectores y de riesgo en Salud Mental en niños y adolescentes de la ciudad de Calama. Revista chilena de pediatría, 75(5), 425-433. doi: https://dx.doi.org/10.4067/S037041062004000500003

Hattori, M., Zhang, G., \& Preacher, K. J. (2017). Multiple Local Solutions and Geomin Rotation. Multivariate Behavioral Research, 52, 720-731. doi: 10.1080/00273171.2017.1361312

Hombrados, M. (2011). Sentido de comunidad. En Molero, F., Fernández, I., \& Morales, J. (Eds.), Psicología de la intervención comunitaria. (pp. 97-128). Bilbao: Desclée de Brouwer.

Israelashvili, M. (1997). School adaptation, and school member's future expectations of adolescents. Journal of adolescence, 20, 525-535. doi: 10.1006/jado.1997.0107
Jason, L., Stevens, E., \& Ram, D. (2015). Development of a three-factor psychological sense of community scale. Journal of Community Psychology, 43, 973-985. doi: 10.1002/ jcop. 21726

Long, D., \& Perkins, D. (2003). Confirmatory factor analysis of the sense of community index and development of a brief SCI. Journal of Community Psychology, 31(3), 279-296. doi: 10.1002/jcop.10046

McMillan, D., \& Chavis, D. (1986). Sense of community: A definition and theory. Journal of Community Psychology, 14, 6-23. Recuperado de https://pdfs.semanticscholar.org/ e5fb/8ece108aec36714ee413876e61b0510e7c80.pdf

Moscardino, U., Scrimin, S., Capello, F., \& Altoè, G. (2010). Social Science \& Medicine, 70(1), 27-34. doi: 10.1016/j. socscimed.2009.09.035

Obst, P. L., \& White, K. M. (2004). Revisiting the sense of community index: A confirmatory factor analysis. Journal of community psychology, 32(6), 691-705. doi: https://doi. org/10.1002/jcop.21664

Pereda, C. (2003). Escuela y comunidad: observaciones desde la teoría de sistemas sociales complejos. Revista Electrónica Iberoamericana sobre Calidad, Eficacia y Cambio en Educación, 1(1). Recuperado de http://www.ice.deusto.es/ rinace/reice/volln1/Pereda.pdf

Peterson, N. A., Speer, P. W., \& McMillan, D. W. (2008). Validation of a Brief Sense of Community Scale: Confirmation of the principal theory of sense of community. Journal of Community Psychology, 36, 61-73. doi: 10.1002/jcop.20217

Peterson, N. A., Speer, P. W., \& Hughey, J. (2006). Measuring sense of community: A methodological interpretation of the factor structure debate. Journal of Community Psychology, 34(4), 453-469. doi: 10.1002/jcop.20109

Pretty, G. H., Andrews, L., \& Collett, C. (1994). Exploring adolescents' sense of community and its relationship to loneliness. Journal of Community and Applied Social Psychology, 22, 346-358. doi: 10.1002/1520-6629(199410)22:4<346:AIDJCOP2290220407>3.0.CO;2-J

Puddifoot, J. (1994). Community identity and sense of belonging in a northeastern English town. Journal of social Psychology, 134, 601-608. doi: 10.1080/00224545.1994.9922990

Redding, S. (2001). The Community of the School. En Redding, S., \& Thomas, L. (Eds.), The Community of the School (pp. 1-25). United State of America: The Academic Development Institute.

Rojas-Andrade, R., \& Leiva, L. (2015). Psychopathology and Occasional Victimization in a Sample of Chilean Students. Universitas Psychologica, 14(1), 165-176. doi: http:// dx.doi.org/10.11144/Javeriana.upsy14-1.pvop

Rostosky, S., Owens, G., Zimmerman, R., \& Riggle, E. (2003). Associations among sexual attraction status, school belonging, and alcohol and marijuana use in rural high school students. Journal of Adolescence, 26, 741-751. doi: 10.1016/j. adolescence.2003.09.002 
Sánchez-Vidal, A. (2001). Medida y estructura interna del sentimiento de comunidad: Un estudio empírico. Revista de Psicología Social, 16 (2), 157-175. doi: https://doi. org/10.1174/021347401317351116

Sánchez-Vidal, A. (2009). Validación discriminante de una escala de sentimiento de comunidad. Análisis comparativo de dos comunidades. International Journal of Psychology and Psychological Therapy, 9, 161-176. Recuperado de https:// www.ijpsy.com/volumen9/num2/229/validacin-discriminante-de-una-escala-de-ES.pdf

Sarriera, J. C., Strelhow, M. R., Bedin, L. M., Moura Junior, J. F., Rodrigues, A. L., \& Calza, T. Z. (2015). Adaptation of the Sense of Community Index for Brazilian children. Paidéia (Ribeirão Preto), 25(60), 39-47. doi: 10.1590/198243272560201506

Sayer, E., Beaven, A., Stringer, P., \& Hermena, E. (2013). Investigating sense of community in primary schools. Educational \& Child Psychology, 30(1), 9-25.
Tanaka, J. S. (1987). "How big is big enough?”: Sample size and goodness of fit in structural equation models with latent variables. Child Development, 58(1), 134-146. doi: 10.2307/1130296

Watson, T. N., \& Bogotch, I. (2016). (Re)Imagining School as Community: Lessons Learned from Teachers. School Community Journal, 26(1), 93-114. Recuperado de https://files. eric.ed.gov/fulltext/EJ1104392.pdf

Witten, K., McCreanor, T., \& Kearns, R. (2007). The places of schools in parents' community belonging. New Zealand Geographer, 63, 141-148. doi: 10.1111/j.17457939.2007.00097.x

Wright, S. (2004). Exploring psychological sense of community in living-learning programs and in the University as a whole (tesis doctoral). University of Maryland. Recuperado de http://drum.lib.umd.edu/bitstream/1903/1687/1/umi umd-1657.pdf 